\title{
Les types de besoins de soutien des enseignants débutants et leur perception de l'aide reçue par le biais des différentes mesures de soutien
}

\section{CHRONIQUE • Insertion professionnelle}

\section{Mots-clés}

Insertion professionnelle, besoin de soutien, difficultés, enseignants débutants, programme d'insertion professionnelle, dispositifs de soutien

La problématique de l'entrée dans la profession enseignante fut abordée abondamment depuis les dernières décennies. En effet, les enseignants débutants ont bien souvent une insertion professionnelle (IP) laborieuse empreinte d'embuches, et ce, pour différentes raisons. Les difficultés souvent recensées touchent la grande mobilité professionnelle (Ingersoll, 2012), la gestion de la classe, la gestion des comportements difficiles des élèves, la gestion des relations interpersonnelles avec leurs collègues et les parents des apprenants, ainsi que la fatigue causée par la lourdeur de la charge de travail (Bland, Church et Luo, 2014; Coggins et Diffenbaugh, 2013). Il y a aussi les inquiétudes liées à l'adaptation des interventions aux élèves en difficulté et à l'évaluation des apprentissages et des compétences des élèves (Mukamurera et Tardif, 2016; Potemski et Matlach, 2014). Le manque de soutien administratif, le peu d'occasions de formation continue et le sentiment d'isolement professionnel sont également des difficultés notables ressenties par un bon nombre d'enseignants en insertion professionnelle (Forseille et Raptis, 2016; Headden, 2014).

Pour contrer ces difficultés et limiter les conséquences de ces dernières (troubles psychologiques, attrition professionnelle, etc.), diverses mesures de soutien à l'insertion sont mises en place pour soutenir les enseignants débutants (Bland et al., 2014; Kang et Berliner, 2012). 
La finalité des mesures de soutien est d'améliorer les compétences et la satisfaction des nouveaux enseignants afin qu'ils développent un sentiment d'auto-efficacité positif, décident de rester dans la profession et que cela entraine, par ricochet, une amélioration des apprentissages et de la réussite des élèves (Ingersoll, 2012).

Ces mesures prennent habituellement la forme de programme d'insertion professionnelle (PIP) ou de dispositifs de soutien offerts aux débutants. Un PIP est structuré et comprend plusieurs dispositifs. Ces derniers sont des mesures plus spécifiques comme le mentorat, les trousses d'accueil, les formations pour les nouveaux enseignants, etc. (Leroux et Mukamurera, 2013). Des chercheurs affirment que, pour être efficaces, ces mesures de soutien doivent, entre autres, répondre aux besoins de soutien des enseignants débutants (Kidd, Brown et Fitzallen, 2015; Leroux et Mukemurera, 2013). En outre, une évaluation systématique de l'efficacité des mesures de soutien mises en place est aussi une des conditions d'efficacité de ces programmes (Mukamurera, Martineau, Bouthiette et Ndoreraho, 2013).

Alors que les difficultés liées à l'IP ont été largement étudiées dans les dernières décennies, qu'en est-il des besoins de soutien des nouveaux enseignants? Que savons-nous à ce sujet? Est-ce que les termes «difficultés » et «besoins" renvoient au même concept? Aussi, comment évaluer si les dispositifs mis en place répondent aux besoins de soutien des enseignants débutants si nous ne connaissons pas réellement leurs besoins? Ces questionnements sont à la base de notre projet doctoral.

Bien que plusieurs chercheurs abordent l'importance de connaitre les besoins des enseignants en IP avant de décider des dispositifs de soutien offerts (Kidd et al., 2015; Leroux et Mukamurera, 2013; Mukamurera et al., 2013), nous n’avons recensé aucune étude décrivant les besoins de soutien des enseignants débutants, outre l'enquête de Mukamurera et Martineau conduite en 2013 et dont quelques statistiques descriptives ont déjà été diffusées.

Un certain engouement se fait sentir au Québec pour l'élaboration et la mise en œuvre de PIP depuis la mesure budgétaire allouée par le ministère de l'Éducation pour soutenir les initiatives liées à la mise en place de mesures de soutien pour les enseignants débutants (gouvernement du Québec, 2013). Ainsi, plusieurs commissions scolaires souhaitent instaurer des PIP et, comme la mise en place de dispositifs de soutien coute cher (Ingersoll, 2012), nous croyons qu'il est important d'établir le degré de concordance entre les dispositifs de soutien et les besoins des débutants, ainsi que d'évaluer l'appréciation des enseignants ayant bénéficié de ces dispositifs.

Ces différents constats nous amènent à nous poser cette question : quels sont les types de besoins de soutien ressentis par les enseignants débutants québécois et en quoi les mesures de soutien offertes y répondent-elles?

Ces connaissances permettront d'éclairer les décideurs afin qu'ils instaurent des PIP dont les dispositifs sont complémentaires, répondent à différents besoins de soutien et que les enseignants qui en bénéficient se sentent réellement soutenus. Les résultats de cette recherche pourront également permettre de bonifier la préparation à la transition vers l'IP dès la formation initiale, ce qui semble peu fait à l'heure actuelle (Leroux, Dufour, Portelance, Meunier, Cividini et Carpentier, 2016). 


\section{Références}

Bland, P., Church, E. et Luo, M. (2014). Strategies for attracting and retaining teachers. Administrative Issues Journal: Education, Practice, and Research, 4(1), 9-19. http://dx.doi.org/10.5929/2014.4.1.2

Coggins, C. et Diffenbaugh, P. (2013). Teachers with drive. Educational Leadership, 71(2), 42-45.

Forseille, A. et Raptis, H. (2016). Future teachers clubs and the socialization of pre-service and early career teachers, 19532015. Teaching and Teacher Education, 59, 239-246. http://dx.doi.org/10.1016/j.tate.2016.06.013

Headden, S. (2014). Beginners in the classroom. What the changing demographics of teaching mean for schools, students, and society. Repéré à https://www.carnegiefoundation.org/wp-content/uploads/2014/09/beginners in classroom.pdf

Ingersoll, R.-M. (2012). Beginning teacher induction. What the data tell us. Phi Delta Kappan, 93(8), 47-51. http://dx.doi.org/10.1177/003172171209300811

Kang, S. et Berliner, D. C. (2012). Characteristics of teacher induction programs and turnover rates of beginning teachers. Teacher Educator, 47(4), 268-282. http://dx.doi.org/10.1080/08878730.2012.707758

Kidd, L., Brown, N. et Fitzallen, N. (2015). Beginning teachers' perception or their induction into the teaching profession. Australian Journal of Teacher Education, 40(3), 154-173. http://dx.doi.org/10.14221/ajte.2014v40n3.10

Leroux, M. et Mukamurera, J. (2013). Bénéfices et conditions d'efficacité des programmes d'insertion professionnelle en enseignement : état des connaissances sur le sujet. Formation et profession, 21(1), 13-27. http://dx.doi.org/10.18162/fp.2013.32

Mukamurera, J., Martineau, S., Bouthiette, M. et Ndoreraho, J.-P. (2013). Les programmes d'insertion professionnelle des enseignants dans les commissions scolaires du Québec : portrait et appréciation des acteurs. Éducation et formation, 299, 13-35. Repéré à http://revueeducationformation.be/include/download.php?idRevue=17\&idRes=155

Mukamurera, J. et Tardif, M. (2016). Épanouissement professionnel : entre développement professionnel, satisfaction au travail et intention de persévérance durant les premières années d'enseignement. Dans L. Ria (dir.) Former les enseignants au XXI' siècle. Volume 2 : Professionnalité des enseignants et de leurs formateurs (p. 113-134). Bruxelles : Éditions De Boeck.

Potemski, A. et Matlach, L. (2014). Supporting new teachers: what do we know about effective state induction policies. Washington, DC : American Institutes for Research.

\section{Pour citer cet article}

Carpentier-Bujold, G. (2017). Les types de besoins de soutien des enseignants débutants et leur perception de l'aide reçue par le biais des différentes mesures de soutien. Formation et profession, 25(1), 100-102.

http://dx.doi.org/10.18162/fp.2017.a123 